\title{
Neutrophil Dysfunction and Host Susceptibility to Periodontal Inflammation: Current State of Knowledge
}

\author{
Corneliu Sima • Michael Glogauer
}

Published online: 25 March 2014

(C) Springer International Publishing AG 2014

\begin{abstract}
Normal polymorphonuclear neutrophil (PMN) function is critical for the maintenance of host-biofilm equilibrium and periodontal tissue homeostasis. Mounting evidence suggests that PMNs play important roles in the control of commensal periodontal flora and initiation of resolution following inflammation caused by accumulating subgingival plaque. Quantitative and qualitative alterations of PMNs in bone marrow, blood, periodontal tissues, and gingival crevicular fluid contribute to host-microbial dysbiosis and onset of irreversible loss of clinical attachment around teeth. Recent findings of specific PMN phenotypes associated with different disease states bring us closer to understanding disease activity and addressing chronic, non-resolved, periodontal inflammation to better monitor and predict patient-specific treatment outcomes. The present review addresses the current state of knowledge in PMN biology in the pathogenesis of periodontal inflammation and the onset of periodontitis.
\end{abstract}

Keywords Neutrophil · Inflammation · Gingivitis · Periodontitis $\cdot$ Host-biofilm $\cdot$ Innate immunity

\section{Introduction}

Polymorphonuclear neutrophils (PMNs) are the most abundant leukocytes in humans accounting for $50-70 \%$ of all circulating white blood cells. They are produced by the bone marrow at a rate of approximately $10^{9}$ cells $/ \mathrm{kg}$ per day from common myeloid precursors. A combination of stem cell factors, interleukin (IL)-3, and granulocyte macrophage

C. Sima $\cdot$ M. Glogauer

Matrix Dynamics Group, University of Toronto, Toronto, ON,

Canada

C. Sima $\cdot$ M. Glogauer $(\square)$

Faculty of Dentistry, Department of Periodontology, University of Toronto, Room 221 Fitzgerald Building, 150 College Street, Toronto, ON M5S 3E2, Canada

e-mail: michael.glogauer@utoronto.ca colony-stimulating factor (GM-CSF) is required for commitment to the myeloid lineage [1]. Differentiated PMNs are released into blood as band (incompletely matured) or segmented (mature) neutrophils after a 12-day maturation period. Classically, it was thought that PMNs spend 6-8 h in the circulation before they migrate into the tissues where they can remain for days and then undergo apoptosis and be cleared by macrophages. More recently, several studies have shown that PMNs can circulate in the blood for up to 5 days [2, 3]. In the extravascular compartment, PMNs participate in several critical functions of the innate immune system including phagocytosis and killing of microorganisms, extra-cellular matrix degradation, and post-inflammatory restoration of tissue homeostasis. It is generally accepted that PMN recruitment and microbicidal function are essential for the maintenance of periodontal health. Research has shown that deviations from normal PMN activity, including altered production, release, recruitment, impaired function, or hyper-reactivity with the exception of some forms of reduced superoxide production, are associated with disruption of periodontal tissue homeostasis that results in tissue destruction and loss of clinical attachment [4•]. Furthermore, PMNs play important roles in periodontitis pathogenesis through impaired apoptotic programs and activation of adaptive immunity that leads to failure to resolve inflammation and disease progression.

\section{Neutrophil Physiology in the Periodontium}

Neutrophil Recruitment

Several lines of evidence suggest that PMN recruitment to, and function in the periodontal tissue are essential for maintaining homeostasis. It is estimated that 30,000 PMNs transit through periodontal tissues every minute and that their presence in the gingival crevicular fluid (GCF) is physiological. In fact, $>90 \%$ of GCF cells are PMNs and they form a barrier between the junctional epithelium and the subgingival biofilm 
preventing its apical migration [5]. IL-8 and intercellular adhesion molecule 1 (ICAM-1) gradients in the junctional epithelium mediate PMN migration to the gingival sulcus [6]. In gingival post-capillary venules, activated endothelial cells increase the expression of chemokines (macrophage inflammatory protein 2-alpha, MIP2- $\alpha$ or CXCL2 and IL-8 or CXCL8), selectins (P and -E selectin or CD62P and CD62E), and intercellular adhesion molecules or ICAMs for capture, rolling, and attachment of circulating PMNs. Activated PMNs increase surface expression of chemokine receptors (CXCR1 or CD181, CXCR2 or CD182), selectin ligands (Pselectin glycoprotein ligand 1 or PSGL-1 or CD162 and $\mathrm{SLew}^{\mathrm{x}}$ containing glycoproteins), and $\beta 2$-integrins (lymphocyte function-associated antigen 1 [LFA-1] or CD11a/CD18). The role of commensal periodontal bacteria in PMN recruitment is incompletely understood. Recent evidence suggests that PMN migration into the healthy periodontium is mediated by selective CXCL2 chemokine upregulation in response to non-pathogenic periodontal bacteria [7]. These observations support the current paradigm that chemoattractants can be functionally divided into "intermediate", found at the blood tissue interface (CXCL8, LTB4) and generally produced by endothelial cells, and "end-target" chemoattractants, found in the immediate vicinity of bacteria (formylmethionyl-leucylphenylalanine; fMLP, C5a). It appears that PMNs preferentially respond to the latter group [8•]. It is therefore possible that specific recruitment mechanisms result in different PMN phenotypes in the tissue and that sequential chemokine engagement can also mediate recruitment. Prior to transmigration into gingival tissues, PMNs undergo rolling mediated by P-selectin-PSGL-1 interactions followed by slow rolling and attachment mediated by LFA-1-ICAM-1 interactions. Using a mouse model of acute gingivitis, we have shown that the leukocyte rolling rate $2 \mathrm{~h}$ after proinflammatory stimulation with tumor necrosis factor- $\alpha$ shows a dose-response relationship with the stimulus [9]. Several physiological inhibitors of PMN integrins have been described. Galectin-1, Lipoxin A4, and Resolvin D series interfere with integrin activation by altered transcription, decreased expression of endothelial ligands, and decreased upregulation. Pentraxin 3 (PTX-3), growth differentiation factor 15 , and developmental endothelial locus 1 (del-1) interfere with the interaction between integrins by antagonism of receptor engagement, interception of inside-out signaling, and antagonism of integrin engagement, respectively [10]. At the disease level, Lipoxin A4 was shown to prevent bone loss and mediate bone regeneration in a rabbit model of ligature-induced periodontitis [11], while del-1 was shown to inhibit PMN infiltration and IL-17-mediated alveolar bone loss in aging mice. Further, del-1-deficient mice developed spontaneous periodontitis characterized by PMN infiltration and IL17 expression [12].
Bacterial Killing

It is currently generally believed that periodontitis is a polymicrobial inflammatory disease initiated by bacteria, and that several bacteria are associated with disease; however, which organisms actually initiate it remains unknown. PMNs play key roles in the control of periodontal biofilm composition by the killing and clearance of pathogens. PMNs can eliminate pathogens by phagocytosis and intracellular killing through oxidative and proteolytic means, and by extracellular mechanisms such as degranulation and release of neutrophil extracellular traps (NETs). Phagocytosis is exponentially enhanced by opsonization through IgG and complement protein C3b. Following recognition of opsonized pathogens, the PMN forms phagocytic cups and then phagosomes, which ultimately fuse with lysosomes to form digestive vacuoles (phagolysosomes) where oxidative and proteolytic antimicrobial molecules are released into an acidic milieu. These include lactoferrin, lysozyme, $\beta 2$-microglobulin, MMP 2 and 9 (collagenase and gelatinase), histaminase, heparinase, sialidase, superoxide, hypochlorous acid $(\mathrm{HOCl})$, and peroxynitrate $\left(\mathrm{ONOO}^{-}\right)$. Although GCF PMN can phagocytose bacteria in the gingival crevice, this may not be the predominant mechanism of protection in this particular niche. When exposed to pathogens, PMN employ efficient oxidative means to kill phagocytosed bacteria mainly through production of reactive oxygen species (ROS) and derivatives ( $\mathrm{HOCl}$, $\mathrm{ONOO}^{-}$). However, ROS generation by PMN may not be critical for maintenance of periodontal health because patients with chronic granulomatous disease having PMNs deficient in producing ROS do not have increased susceptibility to periodontitis [13, 14]. Interestingly, it has been shown that patients with a hyperactive ROS response are more susceptible to periodontitis $[15,16]$. Degranulation may be a more powerful mechanism for control of compositional biofilm changes [5]. The relationship between crevicular PMN and commensal periodontal bacteria is poorly defined. It is believed that similar to intestinal bacteria, oral bacteria associated with periodontal health induce immune tolerance and prevent the host immune system from being activated [17]. It is also likely that non-invading commensal bacteria in the gingival sulcus maintain the continuous influx of PMN that contribute to control of subgingival biofilm composition. The shift from in-offensive to pathogenic subgingival biofilms remains poorly understood. Recent evidence suggests that some periodontal bacteria associated with disease such as Porphyromonas gingivalis can influence the pathogenicity of subgingival biofilms by disrupting the host-microbial homeostasis. On the one hand, $P$. gingivalis can stimulate proinflammatory cytokine production by PMNs and alter their apoptosis through upregulation of Triggering Receptor Expressed on Myeloid cells 1 (TREM-1) [18]. On the other hand, it can trigger changes in the composition and amount of commensal 
bacteria ultimately leading to alveolar bone loss [19•]. Therefore, it seems that the persistence of PMNs inside periodontal tissues combined with ineffective bacterial killing through non-oxidative means contributes to disease activity and tissue breakdown in periodontitis.

\section{Neutrophil Extracellular Traps}

NETs are extracellular fibers extruded actively by the PMN as a type of biological "spider's web." NETs are made up of granule and nuclear constituents that entrap and kill bacteria in the extracellular space $[20,21]$. Only about $30 \%$ of transiently resident PMN release NETs with some evidence that only viable cells can produce them. The current hypothesis of NET formation states that the dying neutrophil is characterized by nuclear swelling, chromatin degradation, and extracellular extrusion of large strands of de-condensed nuclear or mitochondrial DNA that carry with them proteins from the cytosol, granules, and histones from the nuclei [22]. NETs entrap bacteria but they do not appear to be bactericidal. Incubation of entrapped Staphylococcus aureus bacteria and Candida albicans blastospores with DNAse did not result in the killing of these pathogens [23]. The presence of NETs is significantly increased in areas of gingivitis compared with healthy gingival tissues.

Initiation of NET formation is dependent on ROS and patients with chronic granulomatous disease cannot form NETs [24]. Upon ROS release, peptidyl arginine deiminase4 is activated. The latter is known to hypercitrullinate the condensed nuclear chromatin promoting chromatin decondensation. PMN elastase translocates to the nucleus where it digests nucleosomal histones assisting chromatin unfolding [25]. Ultimately, the space between inner and outer nuclear membranes enlarges, forming distinct vesicles that fuse with granule membranes resulting in the release of elastase, myeloperoxidase, and LL-37, and co-localization with nuclear chromatin. Disintegration of the nuclear envelope allows for the DNA/histone complex mixed with granular contents to fill the cytoplasmic space. The final stage involves rupture of the PMN cell membrane and extrusion of the DNA/histone/ cathelicidin antimicrobial peptide mix through changes in the actin cytoskeleton and the microtubular complex [21]. Although the role of NETs in pathogenesis of periodontitis is at an early stage of research, the increased DNAse activity in gingival crevicular fluid during 21-day experimental gingivitis would suggest that removal of biofilm or NET-derived DNA is necessary for inflammation resolution and restoration of health. Therefore, it appears that NET formation in periodontal tissues may not be critical for control of biofilm composition because patients with chronic granulomatous disease do not have increased susceptibility to periodontitis, but NET persistence at sites of periodontal inflammation may be associated with failure of resolution and onset of periodontitis.

\section{Resolution of Inflammation}

Resolution of inflammation is a tightly regulated active process that follows successful removal of non-self. It consists of switching off proinflammatory pathways and clearing local tissue debris ultimately leading to complete restoration of homeostasis. Essential signals from front-line PMNs are needed to initiate resolution. Inside the tissues, PMNs coordinate a lipid mediator switch from proinflammatory (prostaglandins, leukotrienes) to pro-resolving arachidonate and omega- 3 fatty acid derivatives (lipoxins, resolving, protectins, and maresins). It is the lipoxins that provide important signals to switch from inflammatory states characterized by PMN infiltration and activation to resolution and return to tissue homeostasis [26••]. Lipoxin A4 is known to inhibit PMN migration and stimulate non-inflammatory recruitment of monocytes and apoptotic PMN phagocytosis by macrophages [27••]. Once it has accomplished its mission, the PMN triggers selfdestroying mechanisms in a non-inflammatory manner initiating apoptosis through sequential activation of caspases 8,9 , 7 , and 3 , and activation of calpains and ubiquitin-proteasome complexes $[28,29]$. This results in destruction of essential cytoplasmic proteins including the ubiquitously present actin, and chromatin inter-nucleosomal degradation in the nucleus. Aged or dying PMN simultaneously expose phosphatidycholine (PC), phosphatidylethanolamine (PE), and phosphatidylserine (PS), oxidized phospholipids, and carbohydrates including fucose and $\mathrm{N}$-acetyl-glucosamine on their surface [30]. Several pattern recognition molecules (PRM) bind some of the apoptotic cell surface markers and act as bridging molecules to enhance phagocytosis of apoptotic PMNs by macrophages. PRM that have been correlated with apoptotic cells include thrombospondin 1, C1q, mannose-binding lectin, and surfactant proteins (SP-A, SPD) with affinity for carbohydrates, and pentraxins CRP, PTX3, and serum amyloid (SAP) that probably bind chromatin released from dying cells. Specific scavenger receptors including scavenger receptors $\mathrm{A}, \mathrm{B}, \mathrm{CD} 36$, and CD68, $\alpha \mathrm{v} \beta 3$ integrin, PS receptor, and complement receptors (CR1, CR3, CR4) will bind to these molecules activating macrophages and dendritic cells to phagocytose and clear dying PMNs from the tissue [31]. Some non-professional phagocytes such as mesangial and epithelial cells may also help in the clearance of apoptotic PMNs. This physiological "start of the end" allows the PMNs to die silently while sending "find me" and "eat me" signals to be cleared without releasing intracellular components that are highly toxic for host tissues. Nevertheless, a late secondary apoptotic phenotype of PMN can be induced by persistence and increase in proinflammatory lipid mediators such as LTB4. This late apoptosis characterized by 
selective leakage of intracellular content, particularly DNA fragments, is distinct from primary necrosis or NET formation but it may lead to secondary necrosis in the absence of efficient resolving mechanisms [32]. The local and newly recruited monocytes become M2-type (anti-inflammatory) macrophages inside the tissue and phagocytose apoptotic PMN and other debris. Ultimately, macrophages drain via lymphatic vessels into the circulation to be cleared in the spleen or undergo apoptosis locally in a process called efferocytosis [33-35].

\section{Neutrophil Pathology in Periodontal Diseases}

Neutropenia

Defective PMN production in the bone marrow results in neutropenia characterized by reduction of the absolute circulating PMN numbers to $<1,500$ cells $/ \mu \mathrm{L}$. Control of endogenous microbiota is significantly impaired at absolute counts of $<500$ cells $/ \mu \mathrm{L}$ and an inability to mount an inflammatory response is seen at counts of $<200$ cells $/ \mu \mathrm{L}$ [34]. Congenital and acquired (autoimmune, HIV-, and cancer therapyassociated) neutropenia increases the incidence and severity of periodontitis in both primary and permanent dentitions [36]. It is considered that an absolute $\mathrm{PMN}$ count of $<1000 \mathrm{PMN} / \mu \mathrm{L}$ increases the risk of gingivitis [37•]. Pathophysiology of neutropenia can be classified into altered bone marrow stem cell development, altered release from the bone marrow, altered distribution of circulating and marginating PMN pools, and decreased survival of circulating PMN. Benign chronic neutropenia characterized by prolonged noncyclic neutropenia is associated with hyperplastic, edematous, fiery-red gingiva, and in some cases with periodontitis [38, 39]. Cyclic neutropenia characterized by periodic oscillations in production and release of mature PMN including a 21-day cycle with severe neutropenia persisting for 3-10 days is consistently associated with gingivitis and periodontitis [40]. Treatment includes human recombinant granulocyte colony stimulating factor (hrGCSF) three times per week with successful increase in PMN numbers. Congenital neutropenia (Kostman syndrome) is an inherited hematological disorder characterized by an arrest of PMN hematopoiesis at promyelocyte/myelocyte stage and absolute counts of $<2000$ cells $/ \mu \mathrm{L}$. Mutations in the ELANE gene coding for PMN elastase have been associated with periodontitis in patients with severe congenital neutropenia [41]. Gingivitis and severe periodontitis are common complications and despite some temporary improvement in PMN counts with granulocyte colony stimulating factor (G-CSF) treatment, patients with congenital neutropenia tend to have persistent gingivitis. Other conditions characterized by neutropenia and associated with periodontitis are Felty syndrome (an uncommon complication of rheumatoid arthritis), lazy leukocyte syndrome manifested by qualitative and quantitative
PMN defects, and agranulocytosis manifested by a decrease or absence of granulocytes and peripheral leukopenia [37•].

Altered Polymorphonuclear Neutrophil (PMN) Release

During Periodontal Inflammation

Release of PMNs from the bone marrow in response to proinflammatory stimuli is a rate-limiting step in the host response to pathogens. Under normal conditions, more than $90 \%$ of all PMNs are in the bone marrow and roughly $50 \%$ of the circulating PMNs are marginating in postcapillary venules throughout the body [1]. Systemic proinflammatory molecules mediate release of PMNs from the bone marrow through increased G-CSF expression. The mechanisms underlying increased G-CSF expression in periodontitis are incompletely understood. However, persistent PMN recruitment at sites of periodontal inflammation has been associated with activation of the IL-23-IL-17 axis [42]. IL-23 stimulates naive CD4 ${ }^{+} \mathrm{T}$ cells to differentiate into $T_{h} 17$ lymphocytes producing IL-17 that further enhances G-CSF production. It is currently believed that G-CSF interferes with the CXCR4-CXCL12 (SDF-1, stromal cell-derived factor 1) axis, a major regulator of PMN retention in the bone marrow. CXCR4 mutations associated with the WHIM syndrome (warts, hypogammaglobulinemia, infections, and myelokathexis) affect the desensitization of CXCR4 upon stimulation with SDF-1, leading to retention of PMN in the bone marrow [43]. In children with WHIM syndrome, periodontitis is associated with premature tooth loss and recurrent superficial infections. Downstream of CXCR4, Rac GTPases play a crucial role in cytoskeletal organization and genetic control of proliferation and survival pathways. Rac2 plays critical roles in PMN homing in the bone marrow and its absence is associated with neutrophilia [44]. Using Rac null mice, we have recently shown that PMN release from the bone marrow in response to localized ligature-induced periodontitis is significantly increased $24 \mathrm{~h}$ after induction in the absence of Rac2 [45]. Rac2 null mice are more susceptible to alveolar bone loss, possibly because of reduced recruitment and function in the periodontium, and increased retention in periodontal microvasculature. Therefore, existing evidence suggests that increased or decreased PMN release from the bone marrow is associated with higher susceptibility to periodontal inflammation.

\section{Altered PMN Recruitment}

Leukocyte adhesion deficiencies (LADs) represent a group of inherited disorders associated with defects in the expression or function of leukocyte adhesion molecules. Specifically in LAD type 1 (LAD-I), there is a deficiency in $\beta 2$ integrins, in LAD type 2 (LAD-II), there is a deficiency in glycosylation of selectin ligands, and in LAD type 3 (LAD-III), there is dysfunction of signaling intermediates affecting integrin 
activation. A fourth type of LAD (LAD-IV) was proposed for Rac2 mutations affecting PMN chemotaxis and margination [46]. All LADs are characterized by neutrophilia in the absence of infection and impaired PMN recruitment into tissues. Other conditions similar to LADs include Chediak-Higashi syndrome associated with mutations of the LYST gene, which encodes for a protein involved in the regulation of lysosomal trafficking and the Papillon-Lefèvre syndrome caused by deficiency in cathepsin C (dipeptidyl peptidase-I), a lysosomal exo-cysteine protease also involved in pro-enzyme activation (cathepsin G, elastase, and proteinase 3). All syndromes characterized by impaired PMN recruitment and function are associated with increased susceptibility for early onset of severe forms of periodontitis affecting both primary and permanent dentitions [10]. The roles of Rac GTPases in PMN recruitment in periodontitis are less well characterized, possibly because Rac mutations (LAD-IV) are less common. However, observations of PMN dysfunction associated with severe infections in patients with $\mathrm{D} 57 \mathrm{~N} R A C 2$ mutation similar to Rac2 null PMN phenotype in mice suggests that Rac2 may play critical roles in PMN function to control subgingival biofilms. We have recently shown that the initial inflammatory response to induced periodontitis was altered in mice with Rac2 null PMNs. Further, the alveolar bone loss was significantly higher than healthy mice with periodontitis [45]. These findings may be relevant for epigenetic changes that alter the expression of Rac2 and therefore increase susceptibility to periodontitis. Recent focus on epigenetic regulation of gene expression in periodontal inflammation points to potentially significant roles of opportunistic pathogens and the environment on PMN function in inflammatory responses [47].

\section{Impaired Pathogen Clearance}

The association between periodontal pathogens and periodontitis was only defined in cross-sectional studies; therefore failing to demonstrate a direct cause-effect relationship. Human PMN from healthy individuals can efficiently phagocytose and kill the traditionally known "periodontal pathogens" Aggregatibacter actinomycetemcomitans, P. gingivalis, Treponema denticolla, and Tannerella forsythia $[48,49]$. However, these so-called pathogens also possess immune evasion mechanisms that impair or delay their phagocytosis and killing by PMN via leukotoxins, gingipains, and fimbriae that ultimately leads to bacterial tissue and intracellular persistence. Lowabundance bacteria with community-wide effects such as $P$. gingivalis are critical for altering host-biofilm symbiosis by acting directly or indirectly on PMN. Porphyromonas gingivalis can impair PMN recruitment by altering coordinated expression of chemokines (IL-8), tumor necrosis factor- $\alpha$, and adhesion molecules (E-selectin) [50••, 51]. These effects are only transient in vivo suggesting that $P$. gingivalis does not block the recruitment of PMN but rather can delay it.
Porphyromonas gingivalis can escape immune clearance through proactive manipulation of leukocyte receptors and complement. The most documented such mechanism is the $P$. gingivalis-induced C5aR-TLR2 crosstalk in macrophages that impairs iNOS-dependent intracellular bacterial killing [52]. Although these and other mechanisms through which $P$. gingivalis and other periodontal pathogens can manipulate the inflammatory response and bactericidal function of innate immune cells are incompletely understood, a recently proposed model that accommodates these concepts is called the polymicrobial synergy and dysbiosis (PSD) model $[51,53]$. This model implies that physiologically compatible organisms aggregate and coexist in a controlled immuno-inflammatory state being proinflammatory and toxic for the tissues, but the host response controls their overgrowth and overt pathogenicity. Consistent with the ecological plaque hypothesis proposed in the early 1990s to explain the microbial etiology of periodontal diseases, the PSD model supports the concept that non-specific accumulation of subgingival biofilms leads to inflammation of gingival tissues but it is the latter that drives the microbial changes associated with irreversible tissue destruction seen in periodontitis [26••, 54]. In fact, gingivitis is a risk factor for periodontitis whereas only certain bacterial coaggregates and their quantitative changes can predict onset of disease [55••, 56].

\section{Diabetes Mellitus}

Diabetes mellitus (DM) seems to modify periodontal tissues in several ways including immunological dysfunction, microvascular alteration, and changes in extracellular matrix. The relative contribution of these mechanisms to the increased susceptibility for chronic periodontitis and rapid disease progression in diabetic patients is unknown. However, regardless of dental plaque index, gingivitis is more prevalent in patients with DM than in healthy individuals suggesting a direct impact of DM on the local immune response to the bacterial biofilm [57-60]. It is well documented that DM, particularly when uncontrolled, leads to impairment of PMN adherence, chemotaxis, and pathogen clearance [61, 62]. Furthermore, similar to LADs, a common finding in patients with DM is neutrophilia [63]. Although PMNs from patients with DM appear to be primed for hyper-responsive superoxide release, their ability to kill bacteria is often paradoxically impaired [9, 64]. These alterations combined with increased expression of leukocyte adhesion molecules may lead to ectopic inflammatory responses and tissue degradation though enzymatic and oxidative mechanisms. Increased leukocyte adhesion molecule expression and gingival microvascular permeability in $\mathrm{DM}$ in the absence of periodontitis suggests an immunevascular priming that predisposes to periodontitis [9]. Therefore, it seems that in DM, PMNs are released in higher numbers from the bone marrow and are primed to respond 
to inflammatory stimuli before reaching the sites of periodontal inflammation. In addition, PMN and microvascular changes associated with DM seem to predispose to altered PMN margination with subsequent degranulation and oxidative stress in the vasculature that contribute to increased susceptibility, severity, and progression of periodontitis.

\section{Impaired Initiation of Resolution}

Mounting evidence that a failure to resolve inflammation is critical to the chronicity of periodontal tissue inflammation is changing the current paradigm that treatment should primarily focus on control of infectious agents. It is becoming evident that innate immune alterations in response to overgrowing subgingival biofilms result in failure to restore tissue homeostasis and host-microbial symbiosis. PMN apoptosis and expression of "eat me signals" lead to macrophage polarization to proresolution phenotypes in the periodontal environment, which is essential for control of commensal biofilm ecology and tissue integrity. Increased PMN survival and decreased apoptosis was reported in GCF and tissue samples from chronic periodontitis patients $[54,65]$. In fact, one study has shown that GCF PMNs from diseased sites show histone citrullination, a change indicative of PMN activation and initial stages of NET formation. Furthermore, many crevicular PMNs are in advanced stages of NET formation [66]. Similarly, in oral rinses from periodontitis patients, there are more viable and fewer apoptotic PMNs, suggesting increased cell survival in disease [67].
Health

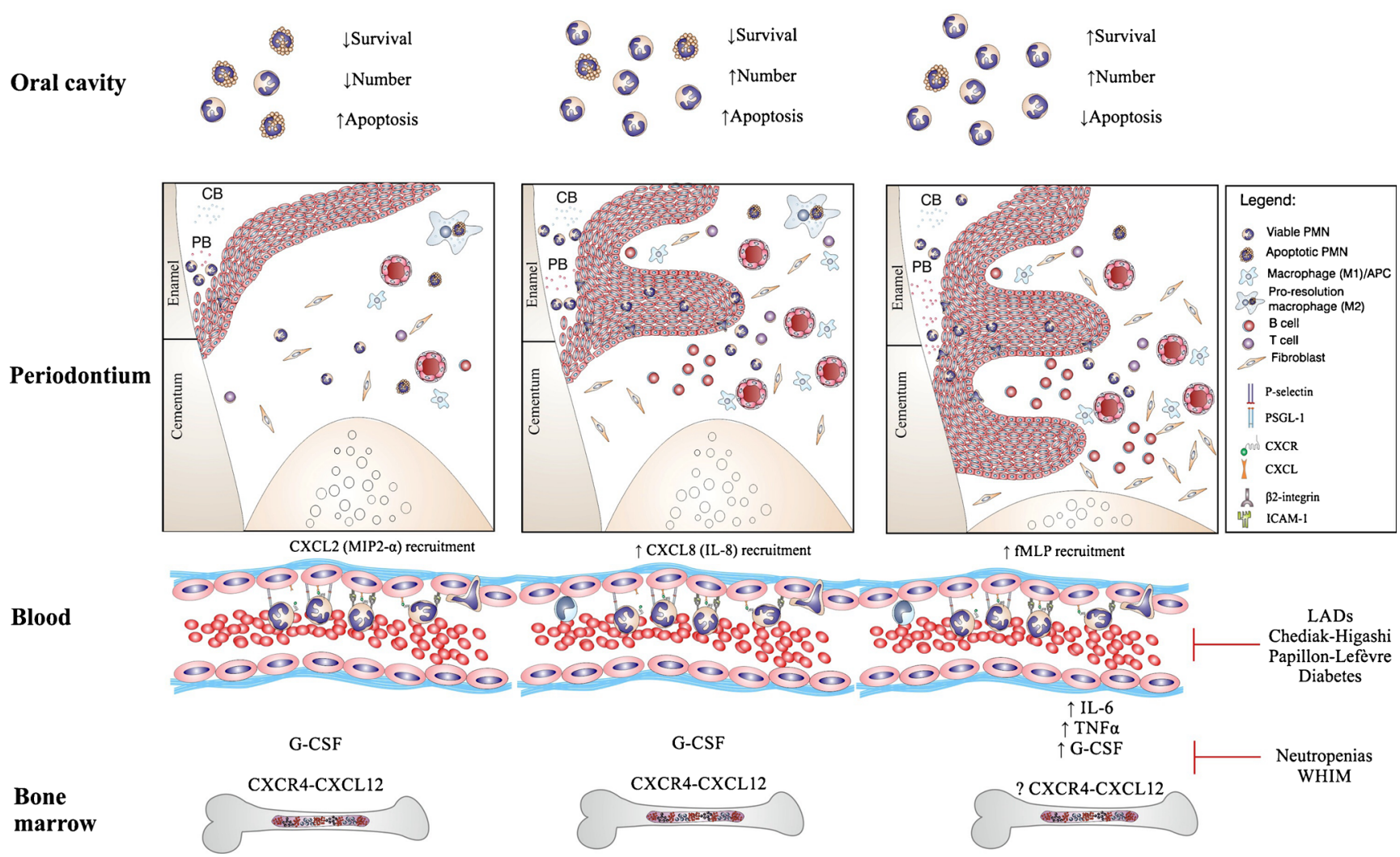

Fig. 1 Polymorphonuclear neutrophil (PMN) recruitment and function in periodontium. In health, persistent low-abundance commensal bacteria in the subgingival biofilm trigger PMN recruitment to the periodontium and $G C F$ gingival crevicular fluid. Recruitment is mediated predominantly by $C X C L 2$ chemokine (C-X-C motif) ligand 2 . In the gingival crevice, PMN form a "wall" between host tissues and the biofilm. Most oral PMNs are short lived or apoptotic and exhibit a downregulation of pro-survival gene expression. In gingivitis, CXCL8-mediated PMN recruitment is predominant; PMN accumulate in higher numbers in the tissue and GCF, particularly in early stages of gingivitis, and have decreased survival and increased apoptosis. In both health and gingivitis inflammation, resolution leads to restoration of homeostasis once PMNs and tissue debris have been cleared by pro-resolution macrophages. In both entities, there is no clinical or histological evidence of alveolar bone loss. In periodontitis (chronic or aggressive forms), local and systemic proinflammatory markers stimulate release of PMNs from the bone marrow. Conditions that impact the release, recruitment, and function of PMNs (e.g., diabetes mellitus, neutropenias, LADs) are associated with different forms of periodontitis. Microbial chemotactic agents such as fMLP, particularly in advanced disease, likely mediate recruitment into periodontal tissues. GCF and oral PMNs have increased survival and decreased apoptosis. $C B$ commensal bacteria, $P B$ pathogenic bacteria, $A P C$ antigen-presenting cell, $f M L P$ formylmethionyl-leucyl-phenylalanine, G-CSF granulocyte colonystimulating factor, $T N F-\alpha$ tumor necrosis factor- $\alpha, M I P 2-\alpha$ macrophage inflammatory protein 2- $\alpha, P S G L-1$ P-selectin glycoprotein ligand 1, ICAM1 intercellular adhesion molecule $1, I L$ interleukin, WHIM warts, hypogammaglobulinemia, infections, and myelokathexis, $L A D s$ leukocyte adhesion deficiencies 
The study of oral PMNs offers several advantages in the case of periodontitis because the subgingival biofilm is located at the interface between periodontal tissues and the oral environment. Therefore, most oral PMN are likely to have encountered the bacterial biofilm, thus offering information on the result of this interaction. The collecting of oral PMNs that extravasate into the mouth through the periodontal crevice was carried out initially by Klinkhammer in the 1960s [68]. Recent work using this approach has demonstrated that PMNs from sites with periodontal disease display increased numbers of gene changes compared with oral neutrophils that enter the healthy periodontium [67]. Genes associated with PMN survival, delayed apoptosis, and PMN recruitment to sites of inflammation were all found to be upregulated in PMNs, which have located to the infected periodontium. This approach has also allowed for the phenotypic characterization of tissue-recruited PMNs. With the identification of novel PMN phenotypes found in tumors, it has been suggested that novel neutrophil subtypes may be associated with some inflammatory diseases $[69,70]$. A recent paper has identified a novel oral PMN subset in healthy patients that expresses the Tcell receptor CD3 [71]. While previous reports suggested that some circulating neutrophils express $\mathrm{CD} 3$ receptors, this work is the first to identify tissue neutrophils expressing T-cell receptors. This finding is significant as it supports the view that PMNs act as a critical bridge between the innate and adaptive immune responses in the context of altered resolution programs. This notion is supported by a well-designed study by Pelletier et al., which demonstrated that activated PMN communicate directly with $\mathrm{T}_{h} 17$ cells by mutual recruitment. PMN also release chemokines, such as CCL20 and CCL2, which have chemotactic activity, and $\mathrm{T}_{\mathrm{h}} 17$ cells release $\mathrm{C}$-XC motif chemokine 8 (CXCL8), which attracts PMN. Using the same approach, they also demonstrated that PMN release CXCL10 and CCL2, which promote chemotaxis of $T_{h} 1$ cells [72]. It therefore seems that increased PMN survival and activity that initiates adaptive immune responses, two indicators of impaired inflammation resolution, are characteristics of active periodontitis.

\section{Conclusions}

The function of PMN from bone marrow to the oral cavity is critical to host-microbial homeostasis and maintenance of periodontal health. Unbalanced (increased or decreased) release, recruitment and function of PMN in the periodontium are likely critical to inflammatory tissue damage, failure of inflammation resolution, and progression from gingivitis to periodontitis. Increasing evidence validates the ecological plaque hypothesis and polymicrobial synergy and dysbiosis theory that account for an altered inflammatory response to accumulating commensal periodontal biofilms as the cause for opportunistic microbial pathogenicity and disease onset (Fig. 1). Genetic and epigenetic factors may contribute to various degrees to altered PMN function associated with loss of periodontal clinical attachment and tooth loss. The heterogeneity in disease expression can be in part explained by the multiple rate-limiting steps in PMN function in relation to periodontal biofilms. Oral PMN characterization throughout the entire spectrum of disease states will further our understanding of inflammation resolution in the periodontium and provide diagnostic tools for monitoring disease activity and response to therapy.

\section{Compliance with Ethics Guidelines}

Conflict of Interest Dr. Corneliu Sima and Dr. Michael Glogauer each declare no potential conflicts of interest relevant to this article.

Human and Animal Rights and Informed Consent This article does not contain any studies with human or animal subjects performed by any of the authors.

\section{References}

Papers of particular interest, published recently, have been highlighted as:

- Of importance

•- Of major importance

1. Summers C, Rankin SM, Condliffe AM, Singh N, Peters AM, Chilvers ER. Neutrophil kinetics in health and disease. Trends Immunol. 2010;31:318-24.

2. Pillay J, den Braber I, Vrisekoop N, Kwast LM, de Boer RJ, Borghans JAM, et al. In vivo labeling with $2 \mathrm{H} 2 \mathrm{O}$ reveals a human neutrophil lifespan of 5.4 days. Blood. 2010;116: 625-7.

3. Bekkering S, Torensma R. Another look at the life of a neutrophil. World J Hematol. 2013;2:44-58.

4. Hajishengallis G. Immunomicrobial pathogenesis of periodontitis: keystones, pathobionts, and host response. Trends Immunol. 2013;35:3-11. This review addresses the host biofilm interations in pathogenesis of periodontitis with focus on inflammation.

5. Delima AJ, Van Dyke TE. Origin and function of the cellular components in gingival crevice fluid. Periodontol 2000. 2003;31:55-7.

6. Tonetti MS, Imboden MA. Neutrophil migration into the gingival sulcus is associated with transepithelial gradients of interleukin- 8 and ICAM-1. J Periodontol. 1998;69:1139-47.

7. Zenobia C, Luo XL, Hashim A, Abe T, Jin L, Chang Y, et al. Commensal bacteria-dependent select expression of CXCL2 contributes to periodontal tissue homeostasis. Cell Microbiol. 2013;15: 1419-26.

8. Kolaczkowska E, Kubes P. Neutrophil recruitment and function in health and inflammation. Nat Rev Immunol. 2013;13:159-75. This review addresses the mechanisms of PMN recruitment and their bactericidal function, including the formation of NETs.

9. Sima C, Rhourida K, Van Dyke TE, Gyurko R. Type 1 diabetes predisposes to enhanced gingival leukocyte 
margination and macromolecule extravasation in vivo. $\mathrm{J}$ Periodontol Res. 2010;45:748-56.

10. Hajishengallis E, Hajishengallis G. Neutrophil homeostasis and periodontal health in children and adults. J Dent Res. 2014;93(3):231-7.

11. Serhan C, Jain A, Marleau S, Clish C, Kantarci A, Behbehani B, et al. Reduced inflammation and tissue damage in transgenic rabbits overexpressing 15-lipoxygenase and endogenous antiinflammatory lipid mediators. J Immunol. 2003;171(12):6856-65.

12. Eskan MA, Jotwani R, Abe T, Chmelar J, Lim JH, Liang S, et al. The leukocyte integrin antagonist Del-1 inhibits IL-17-mediated inflammatory bone loss. Nat Immunol. 2012;13(5):465-73.

13. Martire B, Rondelli R, Soresina A, Pignata C, Broccoletti T, Finocchi A, et al. Clinical features, long-term follow-up and outcome of a large cohort of patients with chronic granulomatous disease: an Italian multicenter study. Clin Immunol. 2008;126:155-64.

14. Cohen MS, Leong PA, Simpson DM. Phagocytic cells in periodontal defense: periodontal status of patients with chronic granulomatous disease of childhood. J Periodontol. 1985;56(10):611-7.

15. Johnstone AM, Koh A, Goldberg MB, Glogauer M. A hyperactive neutrophil phenotype in patients with refractory periodontitis. J Periodontol. 2007;78:1788-94.

16. Aboodi GM, Goldberg MB, Glogauer M. Refractory periodontitis population characterized by a hyperactive oral neutrophil phenotype. J Periodontol. 2011;82:726-33.

17. Feng Z, Weinberg A. Role of bacteria in health and disease of periodontal tissues. Periodontol 2000. 2006;40:50-76.

18. Bostanci N, Thurnheer T, Aduse-Opoku J, Curtis MA, Zinkernagel AS, Belibasakis GN. Porphyromonas gingivalis regulates TREM-1 in human polymorphonuclear neutrophils via its gingipains. PLoS ONE. 2013;8:e75784.

19. Hajishengallis G, Liang S, Payne MA, Hashim A, Jotwani R, Eskan MA, et al. Low-abundance biofilm species orchestrates inflammatory periodontal disease through the commensal microbiota and complement. Cell Host Microbe. 2011;10:497-506. This study demonstrated that an inflammtory disease such as periodontitis can be caused by dysregulation of host-polymicrobial interactions instigated by a single species (Porphyromonas gingivalis) that appears to act as keystone pathogen.

20. Brinkmann V. Neutrophil extracellular traps kill bacteria. Science. 2004;303:1532-5.

21. Cooper PR, Palmer LJ, Chapple ILC. Neutrophil extracellular traps as a new paradigm in innate immunity: friend or foe? Periodontol 2000. 2013;63:165-97.

22. Brinkmann V, Zychlinsky A. Beneficial suicide: why neutrophils die to make NETs. Nat Rev Microbiol. 2007;5:577-82.

23. Menegazzi R, Decleva E, Pietro D. Killing by neutrophil extracellular traps: fact or folklore? Blood. 2012;119:1214-6.

24. Bianchi M, Hakkim A, Brinkmann V, Siler U, Seger RA, Zychlinsky A, et al. Restoration of NET formation by gene therapy in CGD controls aspergillosis. Blood. 2009;114:2619-22.

25. Papayannopoulos V, Metzler KD, Hakkim A, Zychlinsky A. Neutrophil elastase and myeloperoxidase regulate the formation of neutrophil extracellular traps. J Cell Biol. 2010;191:677-91.

26.• Bartold PM, Van Dyke TE. Periodontitis: a host-mediated disruption of microbial homeostasis: unlearning learned concepts. Periodontol 2000. 2013;62:203-17. This paper overviews new concepts in periodontal disease pathogenesis that challenge the existing paradigm of biofilm-initiated tissue destruction.

27.• Serhan CN, Chiang N, Van Dyke TE. Resolving inflammation: dual anti-inflammatory and pro-resolution lipid mediators. Nat Rev Immunol. 2008;8:349-61. This paper is a landmark synthesis of concepts used in analysis of inflammation resolution. It also overviews a new class of endogenously produced pro-resolution lipid mediators.
28. Jesenberger V, Jentsch S. Deadly encounter: ubiquitin meets apoptosis. Nat Rev Mol Cell Biol. 2002;3:112-21.

29. Tait SWG, Green DR. Caspase-independent cell death: leaving the set without the final cut. Oncogene. 2008;27:6452-61.

30. Roos A, Xu W, Castellano G, Nauta AJ, Garred P, Daha MR, et al. Mini-review: a pivotal role for innate immunity in the clearance of apoptotic cells. Eur J Immunol. 2004;34:921-9.

31. Nauta AJ, Daha MR, van Kooten C, Roos A. Recognition and clearance of apoptotic cells: a role for complement and pentraxins. Trends Immunol. 2003;24:148-54.

32. Hébert MJ, Takano T, Holthöfer H, Brady HR. Sequential morphologic events during apoptosis of human neutrophils: modulation by lipoxygenase-derived eicosanoids. J Immunol. 1996;157:3105-15.

33. Bellingan GJ, Caldwell H, Howie SE, Dransfield I, Haslett C. In vivo fate of the inflammatory macrophage during the resolution of inflammation: inflammatory macrophages do not die locally, but emigrate to the draining lymph nodes. J Immunol. 1996; 157:2577-85.

34. Savill JS, Wyllie AH, Henson JE, Walport MJ, Henson PM, Haslett C. Macrophage phagocytosis of aging neutrophils in inflammation: programmed cell death in the neutrophil leads to its recognition by macrophages. J Clin Invest. 1989;83:865-75.

35. Serhan CN, Savill J. Resolution of inflammation: the beginning programs the end. Nat Immunol. 2005;6:1191-7.

36. Hart TC, Atkinson JC. Mendelian forms of periodontitis. Periodontol 2000. 2007;45:95-112.

37. Deas DE, Mackey SA, McDonnell HT. Systemic disease and periodontitis: manifestations of neutrophil dysfunction. Periodontol 2000. 2003;32:82-104.

38. Zaromb A, Chamberlain D, Schoor R, Almas K, Blei F. Periodontitis as a manifestation of chronic benign neutropenia. J Periodontol. 2006;77:1921-6.

39. Armitage GC, Cullinan MP. Comparison of the clinical features of chronic and aggressive periodontitis. Periodontol 2000. 2010;53:12-27.

40. Morley AA, Carew JP, Baikie AG. Familial cyclical neutropenia. Br J Haematol. 1967;13:719-38.

41. Ye Y, Carlsson G, Wondimu B, Fahlén A, Karlsson-Sjöberg J, Andersson M, et al. Mutations in the ELANE gene are associated with development of periodontitis in patients with severe congenital neutropenia. J Clin Immunol. 2011;31:936-45.

42. Gaffen SL, Hajishengallis G. A new inflammatory cytokine on the block: re-thinking periodontal disease and the Th1/Th2 paradigm in the context of Th17 cells and IL-17. J Dent Res. 2008;87(9):817-28.

43. Lagane B, Chow KYC, Balabanian K, Levoye A, Harriague J, Planchenault $\mathrm{T}$, et al. CXCR4 dimerization and beta-arrestinmediated signaling account for the enhanced chemotaxis to CXCL12 in WHIM syndrome. Blood. 2008;112:34- 44.

44. Cancelas JA, Jansen M, Williams DA. The role of chemokine activation of Rac GTPases in hematopoietic stem cell marrow homing, retention, and peripheral mobilization. Exp Hematol. 2006;34:976-85.

45. Sima C, Gastfreund S, Sun C, Glogauer M. Rac-null leukocytes are associated with increased inflammation-mediated alveolar bone loss. Am J Pathol. 2013;184:472-82.

46. Pai S-Y, Kim C, Williams DA. Rac GTPases in human diseases. Dis Markers. 2010;29:177-87.

47. Barros SP, Offenbacher S. Modifiable risk factors in periodontal disease. Periodontol 2000. 2013;64:95-110.

48. Ryder MI. Comparison of neutrophil functions in aggressive and chronic periodontitis. Periodontol 2000. 2010;53:124-37.

49. Eick S, Pfister W, Sigusch B, Straube E. Phagocytosis of periodontopathogenic bacteria by crevicular granulocytes is depressed in progressive periodontitis. Infection. 2000;28:301-4. 
50.• Darveau RP. Periodontitis: a polymicrobial disruption of host homeostasis. Nat Rev Microbiol. 2010;8:481-90. This review integrates recent findings in periodontal microbiology and immunology into contemporary views of periodontitis pathogenesis.

51. Hajishengallis G, Lamont RJ. Beyond the red complex and into more complexity: the polymicrobial synergy and dysbiosis (PSD) model of periodontal disease etiology. Mol Oral Microbiol. 2012;27:409-19.

52. Wang M, Krauss JL, Domon H, Hosur KB, Liang S, Magotti P, et al. Microbial hijacking of complement-toll-like receptor crosstalk. Sci Signal. 2010;3:ra11.

53. Hajishengallis G, Lamont RJ. Breaking bad: manipulation of the host response by Porphyromonas gingivalis. Eur J Immunol. 2014;44(2):328-38.

54. Lucas H, Bartold PM, Dharmapatni AASSK, Holding CA, Haynes DR. Inhibition of apoptosis in periodontitis. J Dent Res. 2009;89: 29-33.

55.• Lang NP, Schätzle MA, Löe H. Gingivitis as a risk factor in periodontal disease. J Clin Periodontol. 2009;36:3-8. This work provides proof-of-concept evidence that gingival inflammation is a risk factor for periodontitis.

56. Charalampakis G, Dahlén G, Carlén A, Leonhardt Å. Bacterial markers vs. clinical markers to predict progression of chronic periodontitis: a 2-yr prospective observational study. Eur J Oral Sci. 2013;121:394-402.

57. Lalla E, Cheng B, Lal S, Kaplan S, Softness B, Greenberg E, et al. Diabetes mellitus promotes periodontal destruction in children. J Clin Periodontol. 2007;34:294-8.

58. Mattout C, Bourgeois D, Bouchard P. Type 2 diabetes and periodontal indicators: epidemiology in France 2002-2003. J Periodontol Res. 2006;41:253-8.

59. Novak KF, Taylor GW, Dawson DR, Ferguson JE, Novak MJ. Periodontitis and gestational diabetes mellitus: exploring the link in NHANES III. J Public Health Dent. 2006;66:163-8.

60. Sarelius IH. Macromolecule permeability of in situ and excised rodent skeletal muscle arterioles and venules. Am J Physiol Heart Circ Physiol. 2005;290:H474-80.
61. Manouchehr-Pour M, Spagnuolo PJ, Rodman HM, Bissada NF. Impaired neutrophil chemotaxis in diabetic patients with severe periodontitis. J Dent Res. 1981;60:729-30.

62. McMullen JA, Van Dyke TE, Horoszewicz HU, Genco RJ. Neutrophil chemotaxis in individuals with advanced periodontal disease and a genetic predisposition to diabetes mellitus. J Periodontol. 1981;52:167-73.

63. Gkrania-Klotsas E, Ye Z, Cooper AJ, Sharp SJ, Luben R, Biggs ML, et al. Differential white blood cell count and type 2 diabetes: systematic review and meta-analysis of cross-sectional and prospective studies. PLoS ONE. 2010;5:e13405.

64. Gyurko R, Siqueira CC, Caldon N, Gao L, Kantarci A, Van Dyke TE. Chronic hyperglycemia predisposes to exaggerated inflammatory response and leukocyte dysfunction in Akita mice. J Immunol. 2006;177:7250-6.

65. Gamonal J, Sanz M, O'Connor A, Acevedo A, Suarez I, Sanz A, et al. Delayed neutrophil apoptosis in chronic periodontitis patients. J Clin Periodontol. 2003;30:616-23.

66. Vitkov L, Klappacher M, Hannig M, Krautgartner WD. Neutrophil fate in gingival crevicular fluid. Ultrastruct Pathol. 2010;34:25-30.

67. Lakschevitz FS, Aboodi GM, Glogauer M. Oral neutrophil transcriptome changes result in a pro-survival phenotype in periodontal diseases. PLoS ONE. 2013;8:e68983.

68. Klinkhamer JM. Quantitative evaluation of gingivitis and periodontal disease: I. The orogranulocytic migratory rate. Periodontics. 1968;6:207-11.

69. Bird L. Tumour immunology: neutrophil plasticity. Nat Rev Immunol. 2009;9:672-3.

70. Fridlender ZG, Sun J, Kim S, Kapoor V, Cheng G, Ling L, et al. Polarization of tumor-associated neutrophil phenotype by TGF- $\beta$ : "N1" versus 'N2' TAN. Cancer Cell. 2009;16:183-94.

71. Lakschevitz FS, Aboodi GM, Glogauer M. Oral neutrophils display a site-specific phenotype characterized by expression of T-cell receptors. J Periodontol. 2013;84:1493-503.

72. Pelletier M, Maggi L, Micheletti A, Lazzeri E, Tamassia N, Costantini C, et al. Evidence for a cross-talk between human neutrophils and Th17 cells. Blood. 2010;115:335-43. 